\title{
Activity of bacteria, mammalian cells, yeasts, cyanobacteria and alga determined by an electrochemical sensor: overview of different applications
}

\author{
Michael Pescheck ${ }^{1}$, Anne Lämmel' ${ }^{2}$ Luděk Bláha ${ }^{1}$ \\ ${ }^{1}$ Masaryk University, Faculty of Science, RECETOX, Kamenice 753/5, 62500 Brno, Czech Republic; \\ ${ }^{2}$ Hochschule Trier, Umwelt-Campus Birkenfeld, Campusallee, 55768 Neubrücke (Nahe), Germany \\ Corresponding author: Michael Pescheck, 390213@mail.muni.cz
}

\begin{abstract}
An electrochemical in vitro sensor system is presented that reflects directly the metabolic activity of prokaryotic and eukaryotic cells. It can be used during a cultivation process to detect growth parameter, as a quality measurement system in food industry and also for the determination of toxic effects of chemicals on bacteria, vertebrate cells, cyanobacteria and algae. The evaluation is based on current-time curves of a potentiostatic measurement produced by the detection of the microbially reduced mediator molecule immobilised in a gel structure. Depending on the number of reduced mediator molecules a current signal is produced, which provides fast information about the viability of living cells. Different senor setups were developed depending on the target application. The final results of the electrochemical sensor show a good correlation with results from reference methods and also with values found in literature.
\end{abstract}

Key words: electrochemical sensor, mediator, activity control, bioactivity monitoring, toxicity testing.

\section{The measurement Principle}

The basic measurement application for the presented biosensor is an amperometric measurement system consisting of a reference electrode, counter-electrode and working electrode (three-electrode system). Mediators are applied in electrochemical investigations of microorganisms to facilitate the transfer of electrons [1], because there is only limited direct transfer of electrons from microorganisms to the electrode of the measurement system [2]. The mediator molecules are reduced rapidly in competition with the electron donors of the organisms and finally transferred to the working electrode surface. This reduced mediator can be recorded at the working electrode of the sensor system as a change in the current. Only living organisms are able to reduce the mediator.

The current measured is direct proportional to the concentration of the reduced mediator, and it is then possible to calculate a metabolic activity of the organism or cell used in the system.
In mammalian cells and yeast cells the mediator 2,3,5,6-tetramethyl-p-benzoquinone (duroquinone, Fig.1) was successfully used. It has structural similarities to ubiquinone, a central molecule in the cell pathway and was well accepted by all cells tested.

2-hydroxy-1,4- naphthoquinone [3] (Fig.2) and anthraquinone were mostly used as a mediator in studies with bacteria and cyanobacteria.

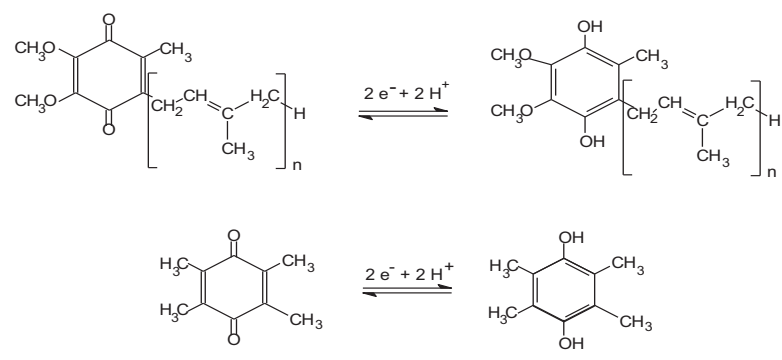

Fig. 1: Structures of oxidised and reduced coenzyme $Q$ - ubiquinone (upper panel), and oxidised and reduced duroquinone (lower panel) [4]. 


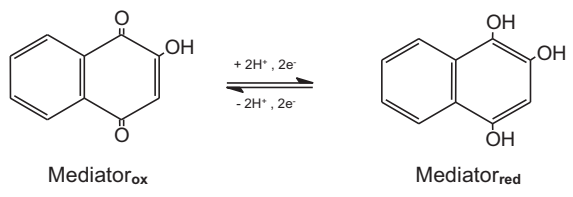

Fig. 2: Oxidised and reduced mediator 2-Hydroxy1,4- naphthoquinone [5].

There are two possibilities to analyze the microbial activity with the presented sensor. Both were based on a current-time curve of the potentiostatic measurement produced by the biologically reduced mediator.

First, it was found that the time to reach the minimum of the recorded current-time curve (Fig. 3) was a useful parameter to describe the biological activity [4].

Second parameter is the slope of the linear part of the current-time curves (Fig. 4). After passing through the minimum of the curve the course of the electric signal showed a steady increase as a function of the metabolic activity. It can directly be described as the slope of the curve [6].

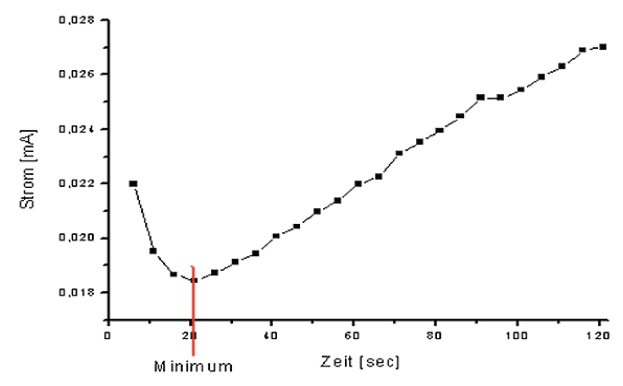

Fig.3: The marked minimum of the current-time curve is reached at a point of time specific to activity of the measured biological culture [4].

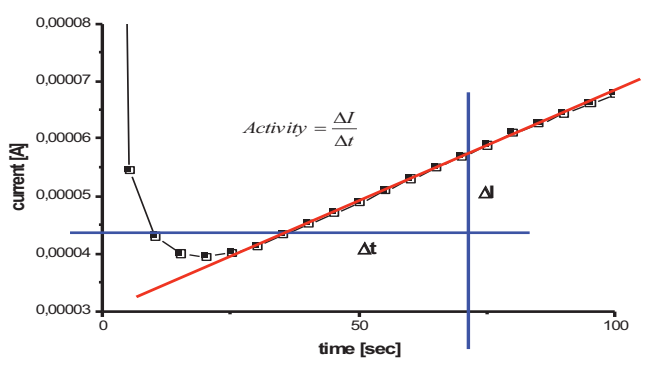

Fig. 4: Typical current-time curve. The slope of the increasing part of the curve directly reflects the metabolic activity of the biological culture [7]

In order to receive a stable and reproducible measurement signal the cells and mediator were immobilised in a Sephadex ${ }^{\mathrm{TM}}-\mathrm{G} 200$ superfine gel (see Fig. 4).
As an advantage both the cells and the mediator are well distributed and fixed in the reaction vessel and the gel structure can also enclose and fix powdery lipophilic mediators without additional solubiliser.

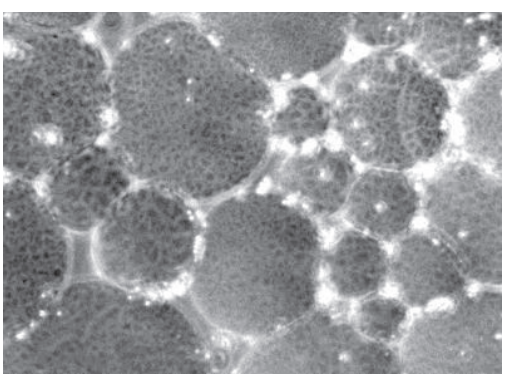

Fig. 4: Cells confined in the interstices of swollen Sephadex ${ }^{T M}$ globules [4].

\section{The measurement cell designs}

During the studies different types of measurement cells were used for the investigations. The beginning was a small vessel construction (Fig.5). The working and counter electrodes of the measuring cell for the first studies with bacteria and yeasts were designed as cylindric vessels with a volume of approx. $5 \mathrm{ml}$ using different types of material (platinated titanium, tantalum and high-grade steel).

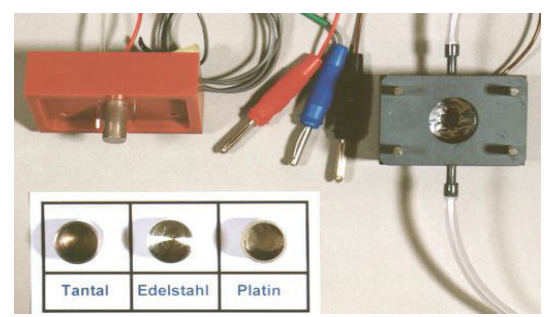

Fig. 5: Picture of the first electrochemical measuring cell tested with the three different counter electrodes [4].

The following construction for mammalian cells was a multiple measurement system with four autonomous reaction measurement cells (vessels, electrodes) designed as spherical elements allowing the detection of four different measuring points at the same time (Fig.6).

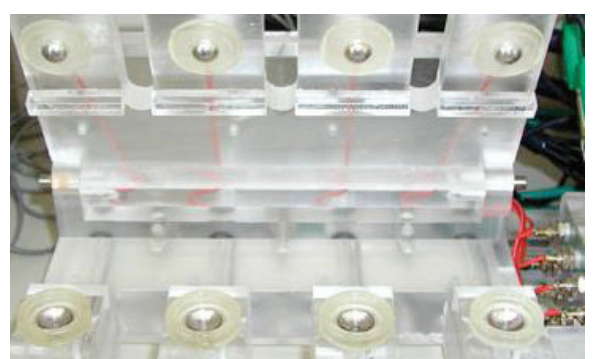

Fig.6: Open measuring system with four individual measurement cells [7] 
The vail volume was reduced to $500 \mu \mathrm{L}$ but the ratio between the vessel volume to electrode surface was increased. The spherical electrodes were made of platinum.

The last modification of the measurement system was created for the application with algae and cyanobacteria (Fig. 7). During the first investigations for these phototrophic organisms 48 well microtiter plates were used, where each row (and each well) could be illuminated separately by LED lights. The platinum electrodes bars together with the reference electrodes were inserted by a special six well electrode holding bar.
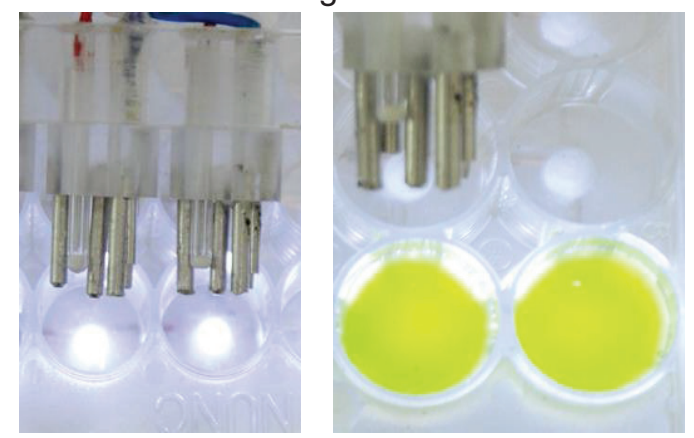

Fig.7: Testing system for algae and cyanobacteria. Left panel - illuminated microtiter plate and the set of electrodes (three working electrode bars, 3 counter electrode bars and one reference electrode); Right panel - wells filled with a mix of $1 \mathrm{ml}$ cyanobacteria culture (Microcystis aeruginosa) and Sephadex G200 gel.

In this case the detection of six different measuring points at the same time can be realized. The vessel volume increased up to 1 $\mathrm{mL}$ using this system.

\section{Results}

\section{Application in food industry}

Starter cultures are used in many applications in the food industry in particular for the fermentative production of foodstuffs and beverages. These cultures can have batch dependent fluctuations in their activities, and this can have adverse effects on the quality of the products. Using the electrochemical measurement cell, all the starter cultures used later in a given food fermentation process can be directly compared with the reference culture to ensure product quality. For our investigations the results obtained with the electrochemical system were compared with established photometric methods (Fig. 8).

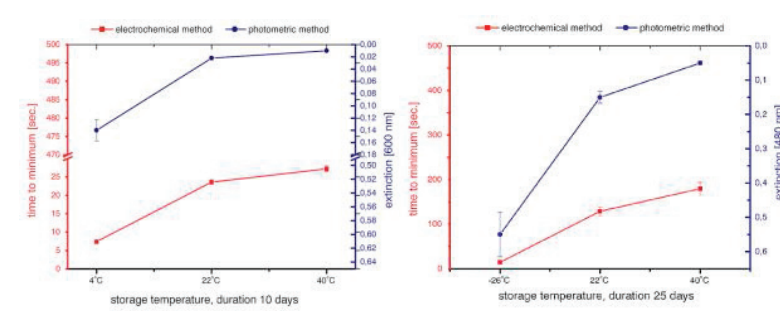

Fig. 8: Application of the sensor in food industry: the activity of the wine yeast SIHA 3 II (left graph), and Staphylococcus carnosus (right graph) [4]. Both experiments compared standard photometric dehydrogenase activity test [8] with the electrochemical measurement cell. The cultures were stored under different temperatures (X-axis) for several days before measurement.

\section{Bioprocess applications}

The electrochemical measurement system has a great potential also in bioprocess control. The results with bacterial strain and yeasts showed that the number of living cells well correlated with the signal of the electrochemical measurement cell (Fig. 9).

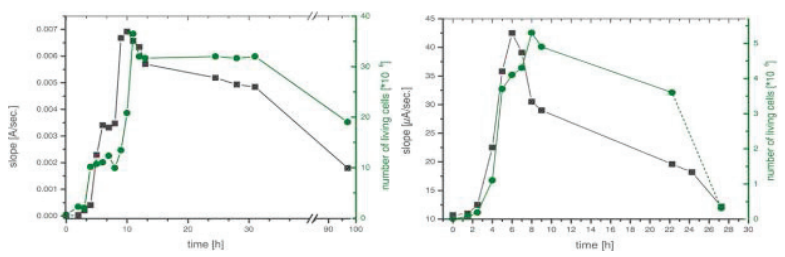

Fig. 9: Comparison of the electrochemical signal (black) with the cell counts (colony forming units green) for Lactobacillus sakei (left graph) and yeast Kluyveromyces marxianus (right graph) [6].

This is an advantage over conventional bioprocess control without sufficient information about living cells or bacteria.

\section{Toxicological applications}

Before marketing of pharmaceuticals, biocides, pesticides and industrial chemicals, an assessment of their potential toxicological and ecotoxicological hazards is required. The interaction of living system with the toxic test substance results in the reduction of biological activity, which is proportional to the toxicity of the studied substance. The electrochemical sensor allowed quick derivation of a specific cytotoxic curve and determination of the corresponding inhibitory concentrations ( $\mathrm{IC}_{50}$ value, Fig. 10). 


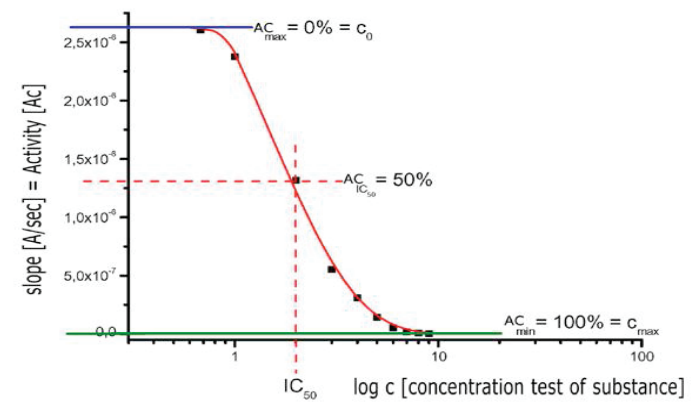

Fig.10 Cytotoxicity curve showing the relationship between the slope of the current/time curves ( $Y$-axis) and the corresponding concentration of the test substance (X-axis, log scale). $A C_{\max }$ is the value of the maximum cell activity when no test substance is added (negative control), $A C_{\min }$ is the value reflecting the complete loss of cell activity. The middle point of the linear part of the curve (IC 50 value) describes the $50 \%$ effect [7].

Tests were performed on mammalian cell lines [7], Fig. 11, and compared with established test methods as well as with literature data.
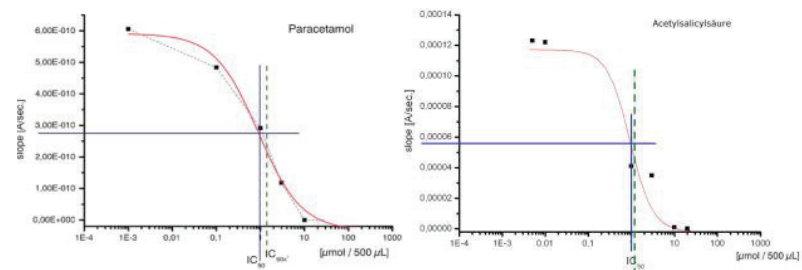

Fig. 11 Cytotoxicity curves of paracetamol (left) acetylsalicylic acid (right) derived using the electrochemical measurement system with $\mathrm{CHO}$ cells. The results of the electrochemical test were a good approximation of those from the literature (blue line: electrochemical test, green dotted lines: literature data [9]).

The pilot results obtained with cyanobacteria and algae are also encouraging. Despite the considerably slower metabolism of the cyanobacteria and algae (as compared with e.g. bacteria or yeasts), current curves were successfully recorded with the new sensor system (data not shown), and possible applications in environmental toxicology are being currently explored.

\section{Conclusion}

The measurements with the presented sensor system allow direct assessment of growth, cell viability or cell activity, and it documents the activity of microorganisms or living cells at any given time.
A significant advantages of the measurement system are easy handling and especially the test time, which is usually much shorter than 1 hour. For bacteria and yeasts few minutes are sufficient to get comprehensive test results.

The electrochemical measurement system can be used successfully in several applications including (i) food industry (assessment of the starter cultures in food fermentation processes), (ii) bioactivity monitoring during bacteria and yeast cultivations, or (iii) toxicity testing with mammalian cells, algae and cyanobacteria.

\section{Acknowledgements}

The authors thank the AiF, Arbeitsgemeinschaft industrieller Forschungsvereinigungen, for the financial support of a great part of this project. Partially supported also by the Czech Ministry of Education (grant No. LO1214). Investigations were also performed at DECHEMA e.V., KarlWinnacker-Institut, Theodor-Heuss-Allee 25, 60486 Frankfurt am Main.

\section{References}

[1] LITHGOW, A.M.; ROMERO, L.; SANCHEZ, I.C.; SOUTO, A.S.; VEGA, C.A.: Interception of the Electron-transport Chain in Bacteria with Hydrophilic Redox Mediators. Part 1. Selective Improvement of the Performance of Biofuel Cells with 2,6-Disulphonated Thionine as Mediator. Journal Chem. Research, 1986, P. 178-179.

[2] FLUTZ, M.L.; DURST, R.A.: Mediator Compounds for the Electrochemical Study of Biological Redox Systems: A Compilation. Analytica Chimica Acta, 140, 1982, P. 1-18.

[3] KREYSA, G.; KRÄMER, P.: Macrokinetics and mathematical modelling on quinone reduction by cyanobacteria. J. Chem. Tech. Biotechnol., 44, 1989, P. 205-217

[4] PESCHECK, M.; SCHRADER, J.; SELL, D.: Characterization of starter cultures by determining microbial activity using an electrochemical gel cell. Food Biotechnol., 16(2), 2002, P. 121- 134

[5] LÄMMEL, A.: Entwicklung eines Sensorsystems zur Messung der biologischen Aktivität von Mikroorganismen und tierischen Zellen. Thesis faculty of Chemistry, 14University Hannover, 1999, P. 14 
[6] PESCHECK, M.; SCHRADER, J.; SELL, D.: Novel electrochemical sensor system for monitoring metabolic activity during the growth and cultivation of prokaryotic and eukaryotic cells. Bioelectrochemistry, 67, 2005, P: 47-55,

[7] PESCHECK, M.; DÜRR, C.; BLÁHA, L.; SELL, $D$.: Novel rapid in-vitro cytotoxicity test on mammalian cells based on an electrochemical measuring method. J Appl Electrochem, 44, 2014, P. 935-943,

[8] Fachgruppe Wasserchemie in der Gesellschaft Deutscher Chemiker, Normenausschuß Wasserwesen im DIN. Bestimmung der Toxizität von Abwässern und Abwasserinhaltsstoffen nach der Dehydrogenasenaktivität mittels 2,3,5Triphenyltetrazoliumchlorid (TTC). In Deutsche Einheitsverfahren zur Wasser-, Abwasser- und Schlammuntersuchung -- Physikalische, chemische, biologische und bakteriologische Verfahren; Wiley-VCH: Weinheim; Vol. V, Part 45, L3, 1999, 1-3

[9] HALLE, W.: Toxizitätsprüfungen in Zellkulturen für eine Vorhersage der akuten Toxizität zur Einsparung von Tierversuchen. BMBF Forschungsvorhaben 0310007A, Jülich. Forschungszentrum, Zentralbibliothek. Schriften des Forschungszentrums Jülich:

Lebenswissenschaften 1, 1998; ISBN 3-89336221-5 